\title{
SYNTHESES, CHARACTERIZATION AND ANTIMICROBIAL STUDIES OF COORDINATION COMPOUNDS OF SCHIFF BASE POSSESSING SEMICARBAZONE MOIETY
}

\author{
D. Kumar*, N. Sharma and S. Chadda \\ Department of Chemistry, National Institute of Technology, \\ Kurukshetra-136119, Haryana (India) \\ *E-mail : dkumar_nitk@yahoo.com
}

\begin{abstract}
Salicylaldehyde and $o$-hydroxyphenylsemicarbazide (OHPSC) react in 1:1 molar ratio in EtOH and produce the Schiff base, $\mathrm{LH}_{4}$ (1). On refluxing a $\mathrm{MeOH}$ solution of 1 with divalent $\mathrm{Mn}(\mathrm{II}), \mathrm{Co}(\mathrm{II}), \mathrm{Ni}(\mathrm{II}), \mathrm{Cu}(\mathrm{II}), \mathrm{Zn}(\mathrm{II}), \mathrm{Cd}(\mathrm{II})$, $\mathrm{Zr}(\mathrm{OH})_{2}(\mathrm{IV}), \mathrm{MoO}_{2}(\mathrm{VI})$ and $\mathrm{UO}_{2}(\mathrm{VI})$ ions in 1:1 molar ratio, produces the corresponding non-electrolytic $\left(\Lambda_{\mathrm{M}}=\right.$ 2.9-10.2 mho cm $\mathrm{mol}^{-1}$ in DMSO) dimeric coordination compounds, $\left[\mathrm{Mn}\left(\mathrm{LH}_{2}\right)\left(\mathrm{H}_{2} \mathrm{O}\right)\right]_{2}(\mathbf{2}),\left[\mathrm{Co}\left(\mathrm{LH}_{2}\right)\left(\mathrm{H}_{2} \mathrm{O}\right)\right]_{2}(\mathbf{3})$, $\left[\mathrm{Ni}\left(\mathrm{LH}_{2}\right)\left(\mathrm{H}_{2} \mathrm{O}\right)\right]_{2}(\mathbf{4}),\left[\mathrm{Cu}\left(\mathrm{LH}_{2}\right)\left(\mathrm{H}_{2} \mathrm{O}\right)\right]_{2}(\mathbf{5}),\left[\mathrm{Zn}\left(\mathrm{LH}_{2}\right)\left(\mathrm{H}_{2} \mathrm{O}\right)\right]_{2}(\mathbf{6}),\left[\mathrm{Cd}\left(\mathrm{LH}_{2}\right)\left(\mathrm{H}_{2} \mathrm{O}\right)\right]_{2}(\mathbf{7}),\left[\mathrm{Zr}(\mathrm{OH})_{2}\left(\mathrm{LH}_{2}\right)\left(\mathrm{H}_{2} \mathrm{O}\right)\right]_{2}(\mathbf{8})$, $\left[\mathrm{MoO}_{2}\left(\mathrm{LH}_{2}\right)\left(\mathrm{H}_{2} \mathrm{O}\right)\right]_{2}(\mathbf{9})$ and $\left[\mathrm{UO}_{2}\left(\mathrm{LH}_{2}\right)\left(\mathrm{H}_{2} \mathrm{O}\right)\right]_{2}(\mathbf{1 0})$ respectively. The various studies like elemental analyses, molar conductance, spectral (IR, reflectance, ${ }^{1} \mathrm{H}$ NMR), TGA and magnetic susceptibility measurements have been used for the characterization of the above synthesized coordination compounds. The Schiff base behaves as a dibasic pentadentate (ONONO donor) ligand and the comparative IR spectral studies of the Schiff base and its coordination compounds suggest the involvement of phenolic $\mathrm{O}$ (aldehydic moiety), azomethine $\mathrm{N}$, carbonyl $\mathrm{O}$, semicarbazidic $\mathrm{N}$ and phenolic $\mathrm{O}$ (aminophenolic moiety) atoms towards coordination. The molecular structures of the Schiff base and its coordination compounds have been optimized by molecular modelling calculations. The Schiff base and all the coordination compounds are good growth retarder for Gram-positive bacteria (S. aureus, B. subtilis), Gram-negative bacteria (E. coli, P. aeruginosa) and yeast (S. cerevisiae, C. albicans). A distorted octahedral structure has been suggested for the coordination compounds.

Keywords: Coordination compounds, distorted octahedral structure, molecular modelling, semicarbazide and spectral studies.
\end{abstract}

(C) RASĀYAN. All rights reserved

\section{INTRODUCTION}

The compounds possessing semicarbazone skeleton are known to exhibit a number of activities namely, anthelmintic, antibacterial, anticonvulsant, antimalarial, antinociceptive, antitubercular, antitumor, fungicidal, lathyrogenic and teratogenic properties. ${ }^{1-4}$ An exhaustive literature survey indicates that comparatively much work has been carried out on the transition metal coordination compounds of Schiff bases containing salicylaldehyde moiety ${ }^{5}$ on the other hand, no work seems to be reported on the coordination compounds of Schiff base derived from salicylaldehyde and OHPSC. Keeping in view, the above mentioned importance of semicarbazide and its derivatives, it was thought worthwhile to synthesize and characterize the Schiff base, $\mathrm{LH}_{4}(\mathbf{1})$ and $\left[\mathrm{Mn}\left(\mathrm{LH}_{2}\right)\left(\mathrm{H}_{2} \mathrm{O}\right)\right]_{2}(\mathbf{2}),\left[\mathrm{Co}\left(\mathrm{LH}_{2}\right)\left(\mathrm{H}_{2} \mathrm{O}\right)\right]_{2}(\mathbf{3})$, $\left[\mathrm{Ni}\left(\mathrm{LH}_{2}\right)\left(\mathrm{H}_{2} \mathrm{O}\right)\right]_{2} \quad(\mathbf{4}), \quad\left[\mathrm{Cu}\left(\mathrm{LH}_{2}\right)\left(\mathrm{H}_{2} \mathrm{O}\right)\right]_{2} \quad(\mathbf{5}), \quad\left[\mathrm{Zn}\left(\mathrm{LH}_{2}\right)\left(\mathrm{H}_{2} \mathrm{O}\right)\right]_{2} \quad(\mathbf{6}), \quad\left[\mathrm{Cd}\left(\mathrm{LH}_{2}\right)\left(\mathrm{H}_{2} \mathrm{O}\right)\right]_{2} \quad$ (7), $\left[\mathrm{Zr}(\mathrm{OH})_{2}\left(\mathrm{LH}_{2}\right)\left(\mathrm{H}_{2} \mathrm{O}\right)\right]_{2}(\mathbf{8}),\left[\mathrm{MoO}_{2}\left(\mathrm{LH}_{2}\right)\left(\mathrm{H}_{2} \mathrm{O}\right)\right]_{2}(\mathbf{9})$ and $\left[\mathrm{UO}_{2}\left(\mathrm{LH}_{2}\right)\left(\mathrm{H}_{2} \mathrm{O}\right)\right]_{2}(\mathbf{1 0})$.

\section{Material and Methods}

\section{EXPERIMENITAL}

All reagents and solvents used were of analytical grade. Hexadecaaqua octahydroxotetrazirconium(IV) acetate and bis(acetylacetonato)dioxomolybdenum(VI) were synthesized according to literature procedures $^{6}$. All the microbial cultures were procured from microbial type culture collection (MTCC), IMTECH, Chandigarh (India). The bacteria were sub-cultured on nutrient agar, whereas the yeasts on

Rasayan J. Chem., 10(2), 391 -398(2017)

http://dx.doi.org/10.7324/RJC.2017.1021643 
malt agar. The molecular structures of the compounds were optimized by CS Chem 3D Pro-14 program package. The methods reported elsewhere, for the estimation of metal contents, spectral studies (IR, reflectance, ${ }^{1} \mathrm{H}$ NMR) and the magnetic susceptibility measurements have been used. ${ }^{7}$ The melting points of compounds were determined on digital melting point apparatus Stuart SMP-40. The molecular weights of the coordination compounds were determined by Rast method using diphenyl as solvent ${ }^{8}$. The molar conductances of coordination compounds in DMSO were carried out using a Toshniwal Conductivity Bridge (Model CL01-02A) and a dip type cell calibrated with $\mathrm{KCl}$ solution. Elemental analyses were carried out using FLASH EA 1112 CHNS (O) analyzer. The IR spectra were recorded in $\mathrm{KBr}(4000-250$ $\mathrm{cm}^{-1}$ ) on a Fourier Transform Infrared Spectrometer Model RZX (Perkin Elmer). The reflectance spectra were recorded on a Hitachi-330 UV-vis-NIR spectrophotometer. ${ }^{1} \mathrm{H}$ NMR spectra were recorded on an Avance-II (Bruker) FT NMR spectrometer at $400 \mathrm{MHz}$, using DMSO as a solvent. The mass spectra were recorded on Waters Micromass Q-Tof Micro-mass spectrometer. The magnetic measurements were carried out at room temperature by Lakeshore VSM 7410 instrument. The TGA was carried out in inert atmosphere, using limiting temperature of $1000{ }^{\circ} \mathrm{C}$ and heating rate of $10{ }^{\circ} \mathrm{C} / \mathrm{min}$. The Agar well diffusion method was used to perform the antimicrobial studies of the ligand (1) and the coordination compounds (2-10) by measuring the MIC and zone of growth inhibition against the test organisms with

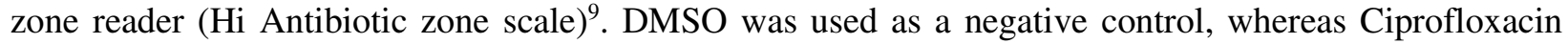
and Amphotericin-B were used as positive and negative control for bacteria and yeast respectively.

\section{General procedure}

Synthesis of OHPSC

To synthesize OHPSC, $o$-aminophenol $(5.45 \mathrm{~g}, 50 \mathrm{mmol})$ was dissolved in $20 \mathrm{~mL}$ of $1: 1 \mathrm{HCl}$. To this solution, semicarbazide hydrochloride $(22.30 \mathrm{~g}, 200 \mathrm{mmol})$ in small quantity was added with constant stirring followed by the addition of distilled water $(30 \mathrm{~mL})$, glacial acetic acid $(1 \mathrm{~mL})$ and concentrated $\mathrm{HCl}(1 \mathrm{~mL})$. The brick red colored solution obtained was boiled under reflux on a water bath for $1 h$. The white crystalline product obtained, was suction filtered, washed with and recrystallized from EtOH and dried over silica gel in vacuo. Yield $=85 \%$, M.p. $=167{ }^{\circ} \mathrm{C}$; anal. $\left[\mathrm{C}_{7} \mathrm{H}_{9} \mathrm{~N}_{3} \mathrm{O}_{2}\right.$; found (calcd.) $\% \mathrm{C}=50.94$ (50.30), $\mathrm{H}=5.47$ (5.39), $\mathrm{N}=25.04$ (25.15); IR bands $\left(\mathrm{cm}^{-1}\right)$ : $3309 \mathrm{v}(\mathrm{O}-\mathrm{H})$ (intramolecular H-bond), 2871 $v(\mathrm{~N}-\mathrm{H})\left(\right.$ intramolecular H-bond), $1687 v(\mathrm{C}=\mathrm{O})$ (carbonyl), $1582 \delta\left(\mathrm{NH}_{2}\right)$ and $1212 v(\mathrm{C}-\mathrm{O})($ enolic-OH$) ;{ }^{1} \mathrm{H}$ NMR (400 MHz; DMSO-d 6 ; $\delta$ ppm): (4H, -ArH, 6.63-7.25), (1H, phenolic-OH, 7.76), $\left(2 \mathrm{H},-\mathrm{NH}_{2}, 5.26\right)$, (2H, $-\mathrm{NH}, 6.42)$.

\section{Synthesis of 1}

To synthesize Sal-OHPSC, salicylaldehyde $(0.61 \mathrm{~g}, 5 \mathrm{mmol})$ and OHPSC $(0.84 \mathrm{~g}, 5 \mathrm{mmol})$ were refluxed in $\mathrm{EtOH}(50 \mathrm{~mL})$ on water bath for $2 \mathrm{~h}$. The cream colored compound was separated out during refluxion. It was suction filtered, washed with and recrystallized from EtOH and then dried as mentioned above. Yield $=90 \%$, M.p. $=201{ }^{\circ} \mathrm{C}$.

\section{Synthesis of 2}

To synthesize coordination compounds $(2)$, a $\mathrm{MeOH}$ solution $(\sim 30 \mathrm{~mL})$ of appropriate metal acetate/compound $(5 \mathrm{mmol})$ and a $\mathrm{MeOH}$ solution $(\sim 50 \mathrm{~mL})$ of $\mathrm{LH}_{4}(0.54 \mathrm{~g}, 5 \mathrm{mmol})$ were boiled under reflux on a water bath for 3-4 $h$. The amorphous solids obtained during refluxion were suction filtered, washed with $\mathrm{MeOH}$ and then dried as mentioned above. Yield $=75-90 \%$.

\section{RESULTS AND DISCUSSION}

The cream colored Schiff base, $\mathrm{LH}_{4}(\mathbf{1})$ (Scheme-1) is formed when an EtOH solution of salicylaldehyde and OHPSC in 1:1 molar ratio is boiled under reflux for $2 \mathrm{~h}$. A MeOH solution of 1 reacts with $\mathrm{MeOH}$ solution of divalent $\mathrm{Mn}(\mathrm{II}), \mathrm{Co}(\mathrm{II}), \mathrm{Ni}(\mathrm{II}), \mathrm{Cu}(\mathrm{II}), \mathrm{Zn}(\mathrm{II}), \mathrm{Cd}(\mathrm{II}), \mathrm{Zr}(\mathrm{OH})_{2}(\mathrm{IV}), \mathrm{MoO}_{2}(\mathrm{VI})$ and $\mathrm{UO}_{2}$ (VI) ions in 1:1 molar ratio and produces the dimeric coordination compounds, $\left[\mathrm{Mn}\left(\mathrm{LH}_{2}\right)\left(\mathrm{H}_{2} \mathrm{O}\right)\right]_{2}(\mathbf{2})$, $\left[\mathrm{Co}\left(\mathrm{LH}_{2}\right)\left(\mathrm{H}_{2} \mathrm{O}\right)\right]_{2}(\mathbf{3}),\left[\mathrm{Ni}\left(\mathrm{LH}_{2}\right)\left(\mathrm{H}_{2} \mathrm{O}\right)\right]_{2}(\mathbf{4}),\left[\mathrm{Cu}\left(\mathrm{LH}_{2}\right)\left(\mathrm{H}_{2} \mathrm{O}\right)\right]_{2}(\mathbf{5}),\left[\mathrm{Zn}\left(\mathrm{LH}_{2}\right)\left(\mathrm{H}_{2} \mathrm{O}\right)\right]_{2}(\mathbf{6}),\left[\mathrm{Cd}\left(\mathrm{LH}_{2}\right)\left(\mathrm{H}_{2} \mathrm{O}\right)\right]_{2}$ (7), $\left[\mathrm{Zr}(\mathrm{OH})_{2}\left(\mathrm{LH}_{2}\right)\left(\mathrm{H}_{2} \mathrm{O}\right)\right]_{2} \quad(\mathbf{8}), \quad\left[\mathrm{MoO}_{2}\left(\mathrm{LH}_{2}\right)\left(\mathrm{H}_{2} \mathrm{O}\right)\right]_{2}$ (9) and $\left[\mathrm{UO}_{2}\left(\mathrm{LH}_{2}\right)\left(\mathrm{H}_{2} \mathrm{O}\right)\right]_{2}$ (10) respectively 
RASĀYAN $J$. Chem.

Vol. 10 | No. 2 |391 -398 | April - June | 2017

(Scheme-2). The coordination compounds are stable towards air and moisture. They get decomposed above $200{ }^{\circ} \mathrm{C}$. The molecular weight measurements in diphenyl indicate their dimeric nature ${ }^{8}$. They are fairly soluble in DMSO but sparingly soluble in DMF, $\mathrm{H}_{2} \mathrm{O}, \mathrm{MeOH}$ and $\mathrm{EtOH}$. Their molar conductance data (Table-1) reveal their non-electrolytic nature. ${ }^{10}$

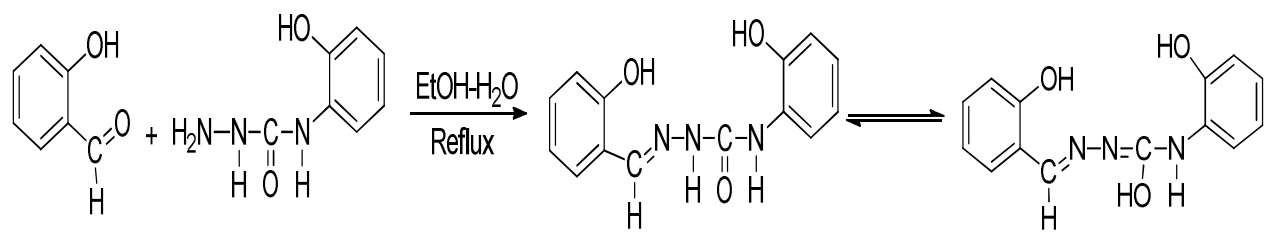

Scheme-1: Synthesis of Schiff base (1)

$$
\begin{aligned}
& 2 \mathrm{LH}_{4}(\mathbf{1})+\mathrm{M}(\mathrm{OAc})_{2} \cdot \mathrm{xH}_{2} \mathrm{O}+2 \mathrm{H}_{2} \mathrm{O} \underset{\text { Reflux }}{\stackrel{\mathrm{MeOH}}{\longrightarrow}}\left[\mathrm{M}\left(\mathrm{LH}_{2}\right)\left(\mathrm{H}_{2} \mathrm{O}\right)\right]_{2}(\mathbf{2 - 7})+2 \mathrm{AcOH}+2 \mathrm{xH}_{2} \mathrm{O} \\
& {[\mathrm{M}=\mathrm{Mn}, \mathrm{Co}, \mathrm{Ni}, \mathrm{x}=4 ; \mathrm{Cu}, \mathrm{x}=1 ; \mathrm{Zn}, \mathrm{Cd}, \mathrm{x}=2]} \\
& 4 \mathrm{LH}_{4}+\left[\mathrm{Zr}_{4}(\mathrm{OH})_{8}\left(\mathrm{H}_{2} \mathrm{O}\right)_{16}\right](\mathrm{OAc})_{8}+2 \mathrm{H}_{2} \mathrm{O} \underset{\operatorname{Reflux}}{\stackrel{\mathrm{MeOH}}{\longrightarrow}} 2\left[\mathrm{Zr}(\mathrm{OH})_{2}\left(\mathrm{LH}_{2}\right)\left(\mathrm{H}_{2} \mathrm{O}\right)\right]_{2}(\mathbf{8})+8 \mathrm{AcOH}+14 \mathrm{H}_{2} \mathrm{O} \\
& 2 \mathrm{LH}_{4}+2\left[\mathrm{MoO}_{2}(\mathrm{acac})_{2}\right]+2 \mathrm{H}_{2} \mathrm{O} \underset{\text { Reflux }}{\stackrel{\mathrm{MeOH}}{\longrightarrow}}\left[\mathrm{MoO}_{2}\left(\mathrm{LH}_{2}\right)\left(\mathrm{H}_{2} \mathrm{O}\right)\right]_{2}(\mathbf{9})+4 \mathrm{acacH} \\
& 2 \mathrm{LH}_{4}+2\left[\mathrm{UO}_{2}(\mathrm{OAc})_{2}\right] \cdot 2 \mathrm{H}_{2} \mathrm{O} \underset{\text { Reflux }}{\stackrel{\mathrm{MeOH}}{\longrightarrow}}\left[\mathrm{UO}_{2}\left(\mathrm{LH}_{2}\right)\left(\mathrm{H}_{2} \mathrm{O}\right)\right]_{2}(\mathbf{1 0})+4 \mathrm{AcOH}+2 \mathrm{H}_{2} \mathrm{O}
\end{aligned}
$$

\begin{tabular}{|c|c|c|c|c|c|c|c|c|}
\hline \multirow[t]{2}{*}{ S.No. } & \multirow[t]{2}{*}{$\begin{array}{l}\text { Molecular } \\
\text { Formula }\end{array}$} & \multirow[t]{2}{*}{ Color } & \multirow[t]{2}{*}{$\Lambda_{\mathrm{M}}$} & \multirow{2}{*}{$\begin{array}{l}\text { M. Wt } \\
\text { Obs. } \\
\text { (Cald.) }\end{array}$} & \multicolumn{4}{|c|}{$\begin{array}{c}\text { Found } \\
(\text { Calcd })(\%)\end{array}$} \\
\hline & & & & & $\mathrm{M}$ & $\mathrm{C}$ & $\mathrm{H}$ & $\mathrm{N}$ \\
\hline 1. & $\mathrm{C}_{14} \mathrm{H}_{13} \mathrm{~N}_{3} \mathrm{O}_{3}$ & Cream & - & $\begin{array}{l}271^{\mathrm{a}} \\
(271)\end{array}$ & - & $\begin{array}{c}61.81 \\
(61.99)\end{array}$ & $\begin{array}{c}4.72 \\
(4.80)\end{array}$ & $\begin{array}{c}15.29 \\
(15.50)\end{array}$ \\
\hline 2. & $\mathrm{Mn}_{2} \mathrm{C}_{28} \mathrm{H}_{26} \mathrm{~N}_{6} \mathrm{O}_{8}(2)$ & Brown & 7.4 & $\begin{array}{c}699^{\mathrm{b}} \\
(683.8)\end{array}$ & $\begin{array}{c}15.95 \\
(16.06)\end{array}$ & $\begin{array}{c}49.28 \\
(49.14)\end{array}$ & $\begin{array}{c}3.70 \\
(3.80)\end{array}$ & $\begin{array}{c}12.43 \\
(12.28)\end{array}$ \\
\hline 3. & $\mathrm{CO}_{2} \mathrm{C}_{28} \mathrm{H}_{26} \mathrm{~N}_{6} \mathrm{O}_{8}(3)$ & $\begin{array}{l}\text { Brick } \\
\text { Red }\end{array}$ & 4.9 & $\begin{array}{c}687^{\mathrm{b}} \\
(691.8)\end{array}$ & $\begin{array}{c}17.20 \\
(17.03)\end{array}$ & $\begin{array}{c}48.73 \\
(48.57)\end{array}$ & $\begin{array}{l}3.63 \\
(3.76)\end{array}$ & $\begin{array}{c}12.26 \\
(12.14)\end{array}$ \\
\hline 4. & $\mathrm{Ni}_{2} \mathrm{C}_{28} \mathrm{H}_{26} \mathrm{~N}_{6} \mathrm{O}_{8}(\mathbf{4})$ & Green & 5.2 & $\begin{array}{c}695^{\mathrm{b}} \\
(691.4)\end{array}$ & $\begin{array}{c}17.05 \\
(16.98)\end{array}$ & $\begin{array}{c}48.34 \\
(48.60)\end{array}$ & $\begin{array}{c}3.69 \\
(3.76)\end{array}$ & $\begin{array}{c}12.02 \\
(12.15)\end{array}$ \\
\hline 5. & $\mathrm{Cu}_{2} \mathrm{C}_{28} \mathrm{H}_{26} \mathrm{~N}_{6} \mathrm{O}_{8}(\mathbf{5})$ & $\begin{array}{l}\text { Dirty } \\
\text { Green }\end{array}$ & 4.8 & $\begin{array}{l}732^{b} \\
(701)\end{array}$ & $\begin{array}{c}18.05 \\
(18.12)\end{array}$ & $\begin{array}{c}47.98 \\
(47.93)\end{array}$ & $\begin{array}{c}3.65 \\
(3.71)\end{array}$ & $\begin{array}{c}12.03 \\
(11.98)\end{array}$ \\
\hline 6. & $\mathrm{Zn}_{2} \mathrm{C}_{28} \mathrm{H}_{26} \mathrm{~N}_{6} \mathrm{O}_{8}(\mathbf{6})$ & Cream & 10.2 & $\begin{array}{c}704.8^{\mathrm{a}} \\
(704.8)\end{array}$ & $\begin{array}{c}18.72 \\
(18.56)\end{array}$ & $\begin{array}{l}47.75 \\
(47.67)\end{array}$ & $\begin{array}{c}3.80 \\
(3.69)\end{array}$ & $\begin{array}{c}11.75 \\
(11.92)\end{array}$ \\
\hline 7. & $\mathrm{Cd}_{2} \mathrm{C}_{28} \mathrm{H}_{26} \mathrm{~N}_{6} \mathrm{O}_{8}(7)$ & Cream & 6.3 & $\begin{array}{c}698^{\mathrm{b}} \\
(798.8)\end{array}$ & $\begin{array}{c}28.38 \\
(28.14)\end{array}$ & $\begin{array}{c}42.28 \\
(42.06)\end{array}$ & $\begin{array}{l}3.35 \\
(3.25)\end{array}$ & $\begin{array}{c}10.65 \\
(10.52)\end{array}$ \\
\hline 8. & $\mathrm{Zr}_{2} \mathrm{C}_{28} \mathrm{H}_{30} \mathrm{~N}_{6} \mathrm{O}_{12}(\mathbf{8})$ & Brown & 4.1 & $812.1^{\mathrm{b}}$ & 22.46 & 40.65 & 3.58 & 10.28 \\
\hline
\end{tabular}

Scheme-2: Synthesis of coordination compounds (2-10)

Table-1: Analytical, color, molar conductance $\left(\mathrm{mho} \mathrm{cm}^{2} \mathrm{~mol}^{-1}\right)$, mass spectral and molecular weight data of Schiff base (1) and its coordination compounds (2-10) 
RASĀYAN $J$. Chem.

Vol. 10 | No. 2 |391 -398 | April - June | 2017

\begin{tabular}{c|l|l|l|c|c|c|c|c}
\hline & & & & $(824.4)$ & $(22.12)$ & $(40.83)$ & $(3.64)$ & $(10.19)$ \\
\hline 9. & $\mathrm{Mo}_{2} \mathrm{C}_{28} \mathrm{H}_{26} \mathrm{~N}_{6} \mathrm{O}_{12}(\mathbf{9})$ & Dirty & 3.4 & $843.2^{\mathrm{b}}$ & 23.46 & 40.36 & 3.22 & 10.56 \\
& & Brown & & $(829.8)$ & $(23.11)$ & $(40.56)$ & $(3.13)$ & $(10.26)$ \\
\hline 10. & $\mathrm{U}_{2} \mathrm{C}_{28} \mathrm{H}_{26} \mathrm{~N}_{6} \mathrm{O}_{12}(\mathbf{1 0})$ & Orange & 2.9 & $\begin{array}{c}1158^{\mathrm{b}} \\
(1114)\end{array}$ & $\begin{array}{c}43.58 \\
(43.99)\end{array}$ & $\begin{array}{c}30.13 \\
(30.16)\end{array}$ & $\begin{array}{c}2.45 \\
(2.33)\end{array}$ & $\begin{array}{c}7.69 \\
(7.54)\end{array}$ \\
\hline
\end{tabular}

Abbreviations: ${ }^{\mathrm{a} M a s s}$ spectral data and ${ }^{\mathrm{b}}$ Rast method data

\section{Infrared spectra}

The important IR spectral peaks $\left(\mathrm{cm}^{-1}\right)$ of the Schiff base (1) and its coordination compounds (2-10), recorded in $\mathrm{KBr}$ are shown in Table-2. The Schiff base (1) exhibits the $v(\mathrm{O}-\mathrm{H})$ (intramolecular H-bond), $v(\mathrm{~N}-\mathrm{H})($ intramolecular $\mathrm{H}$-bond), $v(\mathrm{C}=\mathrm{O})($ carbonyl), $v(\mathrm{C}=\mathrm{N})($ azomethine $), v(\mathrm{C}-\mathrm{O}) \Phi$ and $v(\mathrm{C}-\mathrm{O})($ enolic) stretches at 3278, 3154, 1695, 1622, 1520 and $1200 \mathrm{~cm}^{-1}$, respectively. The $v(\mathrm{C}-\mathrm{O})_{\Phi}$ stretch of $\mathbf{1}$ is expected to occur at higher energy as compared to the $v(\mathrm{C}-\mathrm{O})\left(\right.$ enolic) stretch. ${ }^{11}$ This is due to the delocalization of $\pi$-electrons towards the benzene ring. The $v(\mathrm{C}-\mathrm{O}) \Phi$ stretch of 1 occurs at $1520 \mathrm{~cm}^{-1}$. This band shifts to higher energy by $25-37 \mathrm{~cm}^{-1}$ in $\mathbf{2 - 1 0}$, supporting the involvement of phenolic $\mathrm{O}$ atom towards coordination. ${ }^{12}$ The magnitude of the high positive shift of the $v(\mathrm{C}-\mathrm{O}) \Phi$ stretch indicates the dimeric nature of all coordination compounds ${ }^{13}$. The $v(\mathrm{C}=\mathrm{N})$ azomethine stretch of 1 occurring at 1622 $\mathrm{cm}^{-1}$ undergoes a negative shift by $20-35 \mathrm{~cm}^{-1}$ in $\mathbf{2 - 1 0}$, indicating the involvement of azomethine $\mathrm{N}$ atom towards coordination ${ }^{14}$. This downward shift is expected due to the reduction in electron density in azomethine link on compound formation. ${ }^{13}$

The appearance of a band at $1695 \mathrm{~cm}^{-1}$ and another band at $1200 \mathrm{~cm}^{-1}$ due to the $v(\mathrm{C}=\mathrm{O}$ )(carbonyl) and $v(\mathrm{C}-\mathrm{O})$ (enolic) stretches, respectively in $\mathbf{1}$ evinces the occurrence of both keto and enol forms in it. The $v(\mathrm{C}=\mathrm{O})$ (carbonyl) stretch of 1 decreases by $13-52 \mathrm{~cm}^{-1}$ in the coordination compounds, indicating the involvement of carbonyl $\mathrm{O}$ atom towards coordination ${ }^{15}$. Interestingly, the $v(\mathrm{C}-\mathrm{O})($ enolic) as well as the $v(\mathrm{~N}-\mathrm{H})$ stretches remain at the same energies in $\mathbf{1}$ and 2-10 suggesting the non-involvement of enolic $\mathrm{O}$ and hydrzidic $\mathrm{N}$ atoms towards coordination. ${ }^{16}$

All the coordination compounds exhibit a broad band in the region $3279-3340 \mathrm{~cm}^{-1}$ indicating the presence of coordinated water molecule. ${ }^{17}$ The appearance of new non-ligand bands between $535-556 \mathrm{~cm}^{-1}$ and $462-481 \mathrm{~cm}^{-1}$ due to the $v(\mathrm{M}-\mathrm{O})$ and $v(\mathrm{M}-\mathrm{N})$ stretches, respectively in 2-10 supports the involvement of phenolic $\mathrm{O}$ and azomethine $\mathrm{N}$ atoms towards coordination. ${ }^{18}$ Thus, as discussed above it is suggested that Schiff base act as a dibasic pentadentate ONONO donor.

Table-2: IR spectral data $\left(\mathrm{cm}^{-1}\right)$ of the Schiff base (1) and its coordination compounds (2-10)

\begin{tabular}{c|c|c|c|c|c|c|c}
\hline S.No. & Compound & $\begin{array}{c}v(\mathrm{C}=\mathrm{O}) \\
(\text { carbonyl }\end{array}$ & $\begin{array}{c}v(\mathrm{C}=\mathrm{N}) \\
(\text { azomethine })\end{array}$ & $\begin{array}{c}v(\mathrm{C}-\mathrm{O}) \\
(\text { phenolic })\end{array}$ & $v\left(\mathrm{H}_{2} \mathrm{O}\right)$ & $v(\mathrm{M}-\mathrm{O})$ & $v(\mathrm{M}-\mathrm{N})$ \\
\hline 1. & $\mathbf{1}$ & 1695 & 1622 & 1520 & - & - & - \\
\hline 2. & $\mathbf{2}(\mathrm{M}=\mathrm{Mn})$ & 1667 & 1597 & 1557 & 3295 & 535 & 470 \\
\hline 3. & $\mathbf{3}(\mathrm{M}=\mathrm{Co})$ & 1668 & 1602 & 1546 & 3334 & 535 & 481 \\
\hline 4. & $\mathbf{4}(\mathrm{M}=\mathrm{Ni})$ & 1682 & 1592 & 1545 & 3279 & 556 & 462 \\
\hline 5. & $\mathbf{5}(\mathrm{M}=\mathrm{Cu})$ & 1680 & 1588 & 1548 & 3288 & 550 & 477 \\
\hline 6. & $\mathbf{6}(\mathrm{M}=\mathrm{Zn})$ & 1643 & 1587 & 1552 & 3340 & 545 & 465 \\
\hline 7. & $\mathbf{7}(\mathrm{M}=\mathrm{Cd})$ & 1667 & 1596 & 1557 & 3294 & 538 & 467 \\
\hline 8. & $\mathbf{8}(\mathrm{M}=\mathrm{Zr})$ & 1652 & 1598 & 1554 & 3296 & 542 & 479 \\
\hline 9. & $\mathbf{9}(\mathrm{M}=\mathrm{Mo})$ & 1665 & 1602 & 1557 & 3318 & 552 & 480 \\
\hline 10. & $\mathbf{1 0}(\mathrm{M}=\mathrm{U})$ & 1646 & 1589 & 1547 & 3325 & 548 & 476 \\
\hline
\end{tabular}

\section{Reflectance spectral studies}

$\left[\mathrm{Mn}\left(\mathrm{LH}_{2}\right)\left(\mathrm{H}_{2} \mathrm{O}\right)\right]_{2}$ shows three absorption bands at 15875,21332 and $25828 \mathrm{~cm}^{-1}$ assigned to the ${ }^{6} A_{1 \mathrm{~g}} \rightarrow$ ${ }^{4} T_{\mathrm{lg}}(G)\left(v_{1}\right),{ }^{6} A_{1 \mathrm{~g}} \rightarrow{ }^{4} T_{2 \mathrm{~g}}(G)\left(v_{2}\right)$ and ${ }^{6} A_{1 \mathrm{~g}} \rightarrow{ }^{4} A_{\mathrm{gg}}(G)\left(v_{3}\right)$ transitions, respectively. ${ }^{19}$ The absorption bands 
occurring in $\left[\mathrm{Co}\left(\mathrm{LH}_{2}\right)\left(\mathrm{H}_{2} \mathrm{O}\right)\right]_{2}$ at 9118,13333 and $19417 \mathrm{~cm}^{-1}$ are due to the ${ }^{4} T_{1 \mathrm{~g}}(F) \rightarrow{ }^{4} T_{2 \mathrm{~g}}(F)\left(v_{1}\right),{ }^{4} T_{1 \mathrm{~g}}(F)$ $\rightarrow{ }^{4} A_{2 \mathrm{~g}}(F)\left(v_{2}\right)$ and ${ }^{4} T_{1 \mathrm{~g}}(F) \rightarrow{ }^{4} T_{1 \mathrm{~g}}(P)\left(v_{3}\right)$ transitions, respectively ${ }^{20}$. [Ni( $\left.\left(\mathrm{LH}_{2}\right)\left(\mathrm{H}_{2} \mathrm{O}\right)\right]_{2}$ shows absorption bands at 9180,16600 and $25594 \mathrm{~cm}^{-1}$ due to the ${ }^{3} A_{2 g}(F) \rightarrow{ }^{3} T_{2 \mathrm{~g}}(F)\left(v_{1}\right),{ }^{3} A_{2 \mathrm{~g}}(F) \rightarrow{ }^{3} T_{1 \mathrm{~g}}(F)\left(v_{2}\right)$ and ${ }^{3} A_{2 \mathrm{~g}}(F)$ $\rightarrow{ }^{3} T_{\mathrm{g}}(P)\left(v_{3}\right)$ transitions, respectively. ${ }^{20}\left[\mathrm{Cu}\left(\mathrm{LH}_{2}\right)\left(\mathrm{H}_{2} \mathrm{O}\right)\right]_{2}$ shows bands at $15586 \mathrm{~cm}^{-1}$ and $28188 \mathrm{~cm}^{-1}$, respectively corresponding to ${ }^{2} B_{1 \mathrm{~g}} \rightarrow{ }^{2} A_{1 \mathrm{~g}}$ transition. No spectral bands were found below $10000 \mathrm{~cm}^{-1}$ which supports octahedral geometry. ${ }^{21}$

\section{Mass spectra}

The prominent fragmentation peaks in 1 observed at $\mathrm{m} / \mathrm{z}=271,254,237,151,135,104$ and 91 are tentatively assigned to $[\mathrm{M}]^{+*},[\mathrm{M}-\mathrm{OH}]^{+\bullet},[\mathrm{M}-2 \mathrm{OH}]^{+\bullet}, \quad\left[\mathrm{M}-\mathrm{C}_{7} \mathrm{H}_{6} \mathrm{NO}\right]^{+\bullet},\left[\mathrm{M}-\mathrm{C}_{7} \mathrm{H}_{6} \mathrm{NO}_{2}\right]^{+\bullet},\left[\mathrm{M}-\mathrm{C}_{7} \mathrm{H}_{7} \mathrm{~N}_{2} \mathrm{O}_{3}\right]^{+^{+}}$ and $\left[\mathrm{M}-\mathrm{C}_{8} \mathrm{H}_{8} \mathrm{~N}_{2} \mathrm{O}_{3}\right]^{+\cdot}$ respectively. The prominent fragmentation peaks in $\left[\mathrm{Zn}\left(\mathrm{LH}_{2}\right)\left(\mathrm{H}_{2} \mathrm{O}\right)\right]_{2}(\mathbf{6})$ observed at $\mathrm{m} / \mathrm{z}=704.8,689.8,668.8,648.8,592.8,576.8,511.4,475.4$ and 448.8 are tentatively assigned to [M] $]^{+\bullet}$, $\left[\mathrm{M}-\mathrm{CH}_{3}\right]^{+^{+}}, \quad\left[\mathrm{M}-2 \mathrm{H}_{2} \mathrm{O}\right]^{+\bullet}, \quad\left[\mathrm{M}-\mathrm{C}_{3} \mathrm{H}_{4} \mathrm{O}\right]^{+\bullet}, \quad\left[\mathrm{M}-\mathrm{C}_{5} \mathrm{H}_{8} \mathrm{~N}_{2} \mathrm{O}\right]^{+\bullet}, \quad\left[\mathrm{M}-\mathrm{C}_{5} \mathrm{H}_{8} \mathrm{~N}_{2} \mathrm{O}_{2}\right]^{+\bullet}, \quad\left[\mathrm{M}-\mathrm{ZnC}_{5} \mathrm{H}_{8} \mathrm{~N}_{2} \mathrm{O}_{2}\right]^{+\bullet}, \quad[\mathrm{M}-$ $\left.\mathrm{ZnC}_{5} \mathrm{H}_{12} \mathrm{~N}_{2} \mathrm{O}_{4}\right]^{+\bullet}$ and $\left[\mathrm{M}-\mathrm{C}_{10} \mathrm{H}_{16} \mathrm{~N}_{4} \mathrm{O}_{4}\right]^{+\bullet}$ respectively.

\section{${ }^{1}$ H NMR spectra}

The ${ }^{1} \mathrm{H}$ NMR spectra of 1 and $\left[\mathrm{M}\left(\mathrm{LH}_{2}\right)\left(\mathrm{H}_{2} \mathrm{O}\right)\right]_{2}\left[\mathrm{M}=\mathrm{Zn}, \mathrm{Cd}, \mathrm{Zr}(\mathrm{OH})_{2}, \mathrm{MoO}_{2}, \mathrm{UO}_{2}\right]$ were recorded in DMSO- $\mathrm{d}_{6}$. The chemical shifts $(\delta)$ are expressed in ppm downfield from TMS. ${ }^{22}$ The Schiff base (1) exhibits a multiplet between $\delta 6.6-7.5 \mathrm{ppm}$ due to the aromatic protons, a broad band at $\delta 7.70 \mathrm{ppm}$ due to phenolic proton, a singlet at $\delta 8.14 \mathrm{ppm}$ due to the azomethine protons and a doublet at $\delta 6.3 \mathrm{ppm}$ due to the two N-H protons. The absence of the resonance signals due to the phenolic protons in the compounds indicates the deprotonation of their $\mathrm{OH}$ groups followed by involvement in coordination. The appearance of signals due to the enolic proton and $\mathrm{N}-\mathrm{H}$ protons in $\mathbf{1}$ at same $\delta$ values indicates noninvolvement of these groups towards coordination. The new signal appearing between $\delta 3.44-3.49 \mathrm{ppm}$ is tentatively assigned to the protons of coordinated $\mathrm{H}_{2} \mathrm{O}$ molecules. ${ }^{23}$

\section{Thermal analysis}

$\left[\mathrm{Cd}\left(\mathrm{LH}_{2}\right)\left(\mathrm{H}_{2} \mathrm{O}\right)\right]_{2}$ loses its coordinated $\mathrm{H}_{2} \mathrm{O}$ molecules between $135-143{ }^{\circ} \mathrm{C}$. The organic skeleton is lost around $320^{\circ} \mathrm{C}$. $\mathrm{CdO}$, the final product is obtained above $400{ }^{\circ} \mathrm{C}$.

\section{Magnetic susceptibility measurements}

The value of magnetic moments of $\mathbf{2}, \mathbf{3}, \mathbf{4}, \mathbf{5}$ coordination compounds at room temperature are 5.68, 4.53, 3.65 and 1.46 B.M. respectively, suggesting their octahedral behavior ${ }^{24}$ (Figure-1). The comparative lower values of the magnetic moments indicate that antiferromagnetic exchange is involved in present cases.

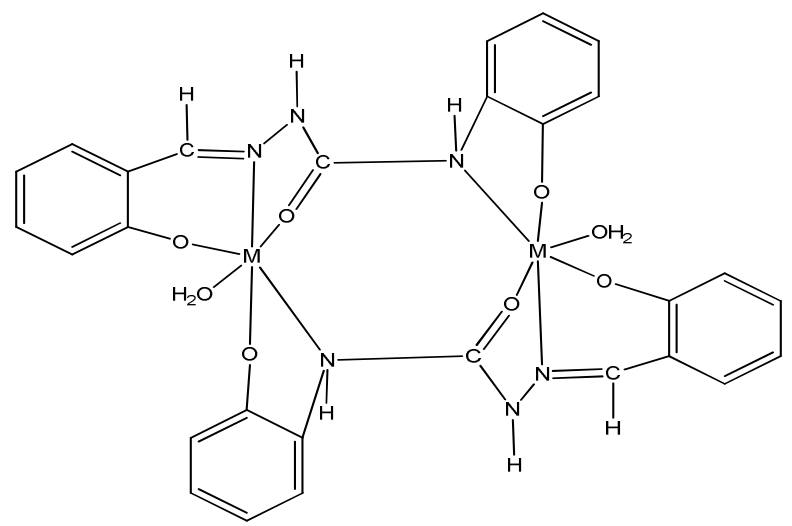

Fig.-1 : Proposed structure of 2-10 (M = Mn, $\left.\mathrm{Co}, \mathrm{Ni}, \mathrm{Cu}, \mathrm{Zn}, \mathrm{Cd}, \mathrm{Zr}(\mathrm{OH})_{2}, \mathrm{MoO}_{2}, \mathrm{UO}_{2}\right)$

\section{Molecular modelling}

The 3D molecular modelling of $\mathbf{1}$ and 2-10 were performed by using MM2 programme contained under CS Chem 3D Pro-14 program package. The 3D optimized geometrical structures of Schiff base and its 
RASĀYAN $J$. Chem.

Vol. 10 | No. 2 |391 -398 | April - June | 2017

coordination compound are presented in Figures-2 and 3. The $[\mathrm{C}(11)=\mathrm{O}(12)$ (carbonyl) bond length,

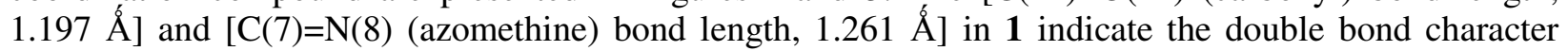
between $\mathrm{C}$ and $\mathrm{O}$ atoms and $\mathrm{C}$ and $\mathrm{N}$ atoms, respectively. ${ }^{25} \mathrm{In}$ coordination compounds, these bond lengths undergo elongation by 0.011-0.089 $\AA$ and 0.012-0.102 $\AA$ respectively. On the other hand, [C(19)$\mathrm{O}(20)$ (phenolic, salicylaldehyde moiety) bond length, $1.365 \AA$ Á] and $\mathrm{C}(5)-\mathrm{O}(9)$ (phenolic, aminophenolic moiety) bond length, $1.361 \AA$ ] of 1 undergo shortening by 0.010-0.085 $\AA$ and 0.013-0.062 $⿱$ Á, respectively (Table-3). The above changes in the respective bond lengths in Schiff base and its coordination compounds are indicative of the coordination of phenolic $\mathrm{O}$ (aldehydic moiety), azomethine $\mathrm{N}$, carbonyl $\mathrm{O}$, semicarbazidic $\mathrm{N}$ and $\mathrm{O}$ (aminophenolic moiety) atoms. ${ }^{26}$ The various bond angles in coordination compounds are indicative of a distorted octahedral geometry. ${ }^{26}$

\section{Antibacterial activities}

Antimicrobial activities of the Schiff base (1) and its corresponding coordination compounds (2-10) were performed against gram-positive bacteria ( $S$. aureus, B. subtilis), gram-negative bacteria (E. coli, $P$. aeruginosa) and fungi (S. cerevisiae, $C$. albicans). Inhibition zone observed around compounds were measured and thus their activities were evaluated. ${ }^{27}$ Positive and negative controls produced significantly sized inhibition zones against the above mentioned bacteria and fungi. The results indicate that all the coordination compounds have higher activity than the Schiff base. $\left[\mathrm{Cd}\left(\mathrm{LH}_{2}\right)\left(\mathrm{H}_{2} \mathrm{O}\right)\right]_{2}$ was the most effective against Gram-positive, Gram-negative bacteria and fungi.

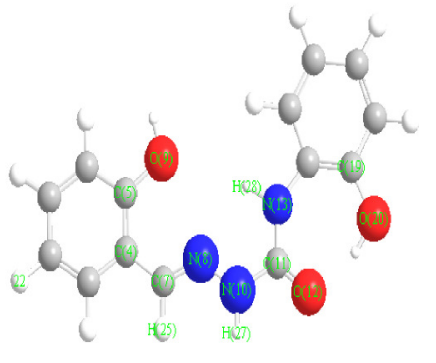

Fig.-2: Energy Optimized structure of Schiff base (1)

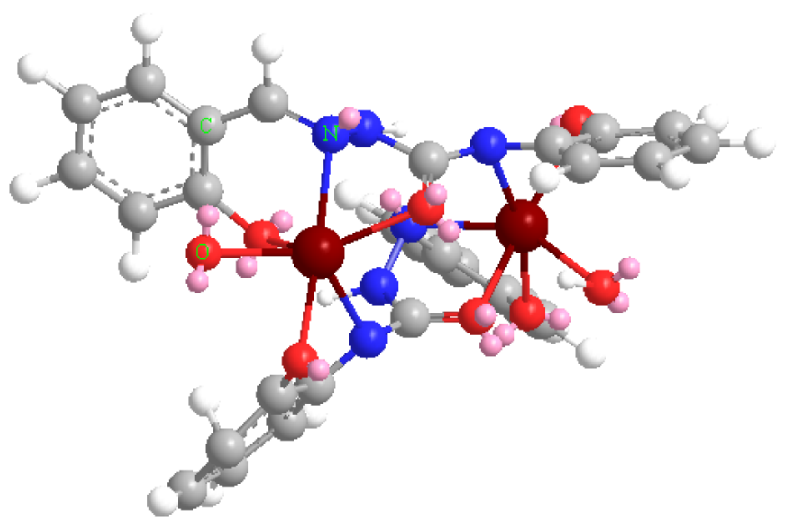

Fig.-3: Energy Optimized structure of 2-10 $(\mathrm{M}=\mathrm{Mn}$, $\left.\mathrm{Co}, \mathrm{Ni}, \mathrm{Cu}, \mathrm{Zn}, \mathrm{Cd}, \mathrm{Zr}(\mathrm{OH})_{2}, \mathrm{MoO}_{2}, \mathrm{UO}_{2}\right)$

Table-3: Bond length $(\AA \hat{)})$ and bond angle $\left({ }^{\circ}\right)$ of the Schiff base $(\mathbf{1})$ and its coordination compounds (2-10)

\begin{tabular}{|c|c|c|c|c|c|c|}
\hline S.No. & Compound & $\begin{array}{l}v(\mathrm{C}=\mathrm{O}) \\
(\text { carbonyl) } \\
(\hat{\AA})\end{array}$ & 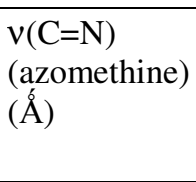 & $\begin{array}{l}\mathrm{v}(\mathrm{C}-\mathrm{O}) \\
(\text { phenolic }) \\
\text { sal moiety } \\
(\hat{A})\end{array}$ & $\begin{array}{l}\mathrm{v}(\mathrm{C}-\mathrm{O}) \\
\text { (phenolic) } \\
\text { aminophenolic } \\
\text { moiety }(\hat{A})\end{array}$ & Bond angle $\left({ }^{\circ}\right)$ \\
\hline 1. & 1 & 1.197 & 1.261 & 1.365 & 1.361 & - \\
\hline 2. & $2(\mathrm{M}=\mathrm{Mn})$ & 1.278 & 1.363 & 1.289 & 1.333 & $\begin{array}{ll}\mathrm{O}_{\text {phenolic }} \mathrm{MnO}_{\text {carbonyl }} & =101.49^{\circ} \\
\mathrm{O}_{\mathrm{H} 2 \mathrm{O}} \mathrm{MnO}_{\text {phenolic }} & =86.35^{\circ} \\
\mathrm{N}_{\text {azomethine }} \mathrm{MnO}_{\text {carbonyl }} & =89.12^{\circ}\end{array}$ \\
\hline 3. & $3(\mathrm{M}=\mathrm{Co})$ & 1.239 & 1.291 & 1.287 & 1.299 & $\begin{array}{ll}\mathrm{O}_{\text {phenolic }} \mathrm{CoO}_{\text {carbonyl }} & =88.40^{\circ} \\
\mathrm{O}_{\mathrm{H} 2 \mathrm{O}} \mathrm{CoO}_{\text {phenolic }} & =87.15^{\circ} \\
\mathrm{N}_{\text {azomethineCoO }} & \mathrm{Coarbonyl}=81.21^{\circ}\end{array}$ \\
\hline 4. & $4(\mathrm{M}=\mathrm{Ni})$ & 1.253 & 1.320 & 1.281 & 1.303 & 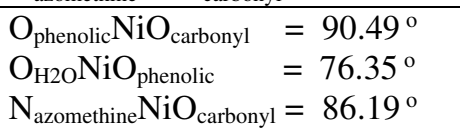 \\
\hline
\end{tabular}


RASĀYAN $J$. Chem.

Vol. 10 | No. 2 |391 -398 | April - June | 2017

\begin{tabular}{|c|c|c|c|c|c|c|}
\hline 5. & $5(\mathrm{M}=\mathrm{Cu})$ & 1.263 & 1.355 & 1.280 & 1.321 & $\begin{array}{ll}\mathrm{O}_{\text {phenolic }} \mathrm{CuO}_{\text {carbonyl }} & =91.40^{\circ} \\
\mathrm{O}_{\mathrm{H} 2 \mathrm{O}} \mathrm{CuO}_{\text {phenolic }} & =99.01^{\circ} \\
\mathrm{N}_{\text {azomethine }} \mathrm{CuO}_{\text {carbonyl }} & =94.21^{\circ}\end{array}$ \\
\hline 6. & $6(\mathrm{M}=\mathrm{Zn})$ & 1.208 & 1.351 & 1.355 & 1.333 & $\begin{aligned} \mathrm{O}_{\text {phenolic }} \mathrm{ZnO}_{\text {carbonyl }} & =91.53^{\circ} \\
\mathrm{O}_{\mathrm{H} 2 \mathrm{O}} \mathrm{ZnO}_{\text {phenolic }} & =85.30^{\circ} \\
\mathrm{N}_{\text {azomethine }} \mathrm{ZnO}_{\text {carbonyl }} & =88.12^{\circ}\end{aligned}$ \\
\hline 7. & $7(\mathrm{M}=\mathrm{Cd})$ & 1.226 & 1.273 & 1.355 & 1.348 & $\begin{array}{ll}\mathrm{O}_{\text {phenolic }} \mathrm{CdO}_{\text {carbonyl }} & =91.49^{\circ} \\
\mathrm{O}_{\mathrm{H} 2 \mathrm{O}} \mathrm{CdO}_{\text {phenolic }} & =86.35^{\circ} \\
\mathrm{N}_{\text {azomethine }} \mathrm{CdO}_{\text {carbonyl }} & =86.13^{\circ}\end{array}$ \\
\hline 8. & $8(\mathrm{M}=\mathrm{Zr})$ & 1.286 & 1.291 & 1.321 & 1.313 & $\begin{array}{ll}\mathrm{O}_{\text {phenolic }} \mathrm{ZrO}_{\text {carbonyl }} & =85.44^{\circ} \\
\mathrm{O}_{\mathrm{H} 2 \mathrm{O}} \mathrm{ZrO}_{\text {phenolic }} & =88.57^{\circ} \\
\mathrm{N}_{\text {azomethine }} \mathrm{ZrO}_{\text {carbonyl }} & =90.37^{\circ}\end{array}$ \\
\hline 9. & $9(\mathrm{M}=\mathrm{Mo})$ & 1.265 & 1.340 & 1.340 & 1.317 & $\begin{aligned} \mathrm{O}_{\text {phenolic }} \mathrm{MoO}_{\text {carbonyl }} & =103.52^{\circ} \\
\mathrm{O}_{\mathrm{H} 2 \mathrm{O}} \mathrm{MoO}_{\text {phenolic }} & =99.36^{\circ} \\
\mathrm{N}_{\text {azomethine }} \mathrm{MoO}_{\text {carbonyl }} & =101.46^{\circ}\end{aligned}$ \\
\hline 10. & $10(\mathrm{M}=\mathrm{U})$ & 1.267 & 1.316 & 1.333 & 1.315 & $\begin{aligned} \mathrm{O}_{\text {phenolic }} \mathrm{CoO}_{\text {carbonyl }} & =101.43^{\circ} \\
\mathrm{O}_{\mathrm{H} 2 \mathrm{O}} \mathrm{CoO}_{\text {phenolic }} & =86.68^{\circ} \\
\mathrm{N}_{\text {azomethine }} \mathrm{CoO}_{\text {carbonyl }} & =98.90^{\circ}\end{aligned}$ \\
\hline
\end{tabular}

\section{CONCLUSION}

It is suggested that $\mathbf{1}$ behaves as the dibasic pentadentate ONONO donor ligand in $\mathbf{2 - 1 0}$ coordinating through its phenolic $\mathrm{O}$ (aldehydic moiety), azomethine $\mathrm{N}$, carbonyl $\mathrm{O}$, semicarbazidic $\mathrm{N}$ and phenolic $\mathrm{O}$ (aminophenolic moiety) atoms. A distorted octahedral structure for 2-10 is proposed. The coordination compounds show significant increased antimicrobial activities as compared to the free Schiff base. Thus, present Schiff base and the corresponding coordination compounds have great potential as antimicrobial agent.

\section{REFERENCES}

1. A.K. Gadad, M.N. Noolvi and R.V. Karpoormath, Bioorg. Med. Chem., 12, 5651(2004).

2. J. Slawinski and M. Gdaniek, Eur. J. Med. Chem., 40, 377(2005).

3. M. Pitucha, Z. Rzaczynska and L. Mazur, X ray Structure Analysis Online, 25, 67(2009).

4. N.K. Sharma, Sapna, M. Agarwal, S. Kohli, B. Tiwari, J.N. Gurtu and B. Agarwal, Orient J. Chem., 26, 103(2010).

5. T.P. Devi and R.K. Hemakumar Singh, Rasayan J. Chem., 3, 266(2010); J. R. Anacona, J. Calvo, O.A. Almanza, Int. J. Inog. Chem., 108740(2013).

6. D. Kumar, P.K. Gupta and A. Syamal, Indian J. Chem., 41, 2494(2002).

7. D. Kumar, A. Syamal, A. Gupta, M. Rani and P.K. Gupta, J. Indian Chem. Soc., 87, 1185 (2010).

8. G. Mann, B. C. Saunders, "Practical Oragnic Chemistry", Longmans, 435, 1961.

9. I. Ahmad and A.Z. Beg, J. Ethnopharm., 74, 113(2001); J.M. Andrews, J. Antimicrob. Chemother., 48, 5(2001); O.H.S. Al-Obaidi, Int. J. Chem. Res., 3, 1(2012).

10. W.G. Geary, Coord. Chem. Rev., 7, 81(1971).

11. A. Syamal, O.P. Singhal, Transition Met. Chem., 4, 179(1979); D. Kumar, N. Sharma, S. Chadda J. Indian Chem. Soc., 93, 1343(2016).

12. R.K. Dubey, U.K. Dubey and C.M. Mishra, Indian J. Chem., 47A, 1208(2008).

13. A. Syamal, J. Indian Chem. Soc., 85, 231(2008).

14. D. Kumar, J. Sharma, S. Chadda and A. Syamal, J. Indian Chem. Soc., 91, 185(2014).

15. J.E. Covacic, Spectrochim. Acta, 23A, 183(1967); B.B. Mahapatra and B.K. Mahapatra, J. Inorg. Nucl. Chem., 39, 2291(1977).

16. A.P. Mishra and M. Soni, Metal-Based Drugs, Article ID 875410, 1(2008).

17. D. Kumar, A. Syamal, A. Gupta, V. Pandey and M. Rani, J. Indian Chem. Soc., 89, 745 (2012). 
18. A. Sharma and M. Shah, J. Appl. Chem., 3, 62(2013); D. Kumar, J. Sharma, S. Chadda and A. Syamal, J. Indian Chem. Soc., 90, 1077(2013).

19. D. Kumar, S. Chadda, J. Sharma and P. Surain, Bioinorg. Chem. Appl., Article ID 981764, 1(2013).

20. A.B.P. Lever, Electronic Spectra of Ions in Inorganic Electronic Spectroscopy, $2^{\text {nd }}$ Ed, Elsevier, Amsterdam, 1984; S. Chandra and U. Kumar, Spectrochim. Acta Part A, 61, 219 (2005); V. Phillip, V. Suni, M.R.P. Kurup and M. Nethaji, Spectrochim. Acta Part A, 64, 171(2006).

21. A. Mumtaz, T. Mahmud and M.R. Elsegood, G.W. Weaver, J. Nucl. Med. Radiat. Ther., 7 (2016).

22. R.M. Silverstein and G.C. Bassler, $2^{\text {nd }}$ ed., Wiley Interscience, New York, 1967.

23. O.B. Ibrahim, M.A. Mohamed and M.S. Refat, Canad. Chem. Trans., 2, 108(2014).

24. R.L. De, M. Mandal, L. Roy, J. Mukherjee, R. Bhawal and K. Maiti, Indian J. Chem., 47, 1480(2008).

25. T.M. Ismail, M.A. EL Gharmy, S.M.A. EL-Wafa and D.F. Sallam, Modern Chemistry., 3, 18(2015).

26. K. Siddappa, S.B. Mane and D. Manikprabhu, The Scient. Wor. J., Article ID 817365, 1 (2014).

27. K.R. Aneja, C. Sharma and R. Joshi, J. Microbio., 4, 175(2011).

[RJC-1643/2017] 